\title{
Candidate SPB and $\gamma$ Doradus stars from microlensing surveys
}

A. Narwid, Z. Kołaczkowski and A. Pigulski

\author{
Astronomical Institute, University of Wrocław, Kopernika 11, 51-622 Wrocław, Poland
}

\begin{abstract}
From the analysis of the database of 200000 variable candidates in the OGLE-II Galactic fields we have extracted about 600 stars showing periodic low-amplitude brightness variations. Many of them are multiperiodic. From their location in the colour-magnitude diagram we conclude that they are good candidates for slowly pulsating B and $\gamma$ Doradus stars.
\end{abstract}

\section{The data and analysis}

Using data from the catalogue of 200000 variable stars candidates we searched short-period low-amplitude pulsators. This photometry was accumulated during the OGLE-II project carried out in the years 1997-2000 in the 49 fields located near the centre of the Galaxy (Woźniak et al. 2002).

The analysis consisted of an automatic extraction of up to five periodic terms for all stars in the catalogue with consecutive prewhitening followed by an automatic classification based upon the periods, amplitudes and Fourier coefficients (for stars with detected harmonics or subharmonics). Then, for stars selected in this way, a detailed analysis was performed in an interactive way. The analysis yielded a lot of candidates for $\beta$ Cephei and $\delta$ Scuti stars (Narwid et al. 2006). In addition, we detected a sample of over 600 low-amplitude variable stars with periods in the range between 0.5 and 6 days. We suspect that the sample consists mainly of a mixture of SPB and $\gamma$ Doradus stars.

For 270 of the variables, we were able to combine the OGLE-II data with the photometry available from the MACHO survey. The analysis of the combined photometry resulted in better resolution, lower detection threshold and practically removed the ambiguity in the frequencies of the detected periodicities. No transformations were performed before data combination.

\section{The results and conclusions}

The results we obtained can be summarized in the following way. The position in the colourmagnitude diagram (CMD) clearly suggests that these stars are main-sequence pulsators. Their $\mathrm{V}$ magnitudes range from 12 to $18 \mathrm{mag}$. The distribution of periods shows that most of these stars have periods in the range between 0.55 and $1.5 \mathrm{~d}$; for periods longer than $2.5 \mathrm{~d}$ the number of variables decreases with increasing period. The I-filter semi-amplitudes of the variables are typically below $20 \mathrm{mmag}$, but there are stars with semi-amplitudes of up to $90 \mathrm{mmag}$. The brightness variability with periods from the aforementioned range is typical for candidate SPB and $\gamma$ Doradus stars. Thus, we suppose that our sample consists mainly of a mixture of these two types of pulsators. This conclusion is supported by the fact that about 400 stars in our sample show multiperiodic behaviour. Up to seven modes in a single star were found in the combined OGLE-II and MACHO data.

In the long-period range contamination from the other types of variability, e.g. $\alpha^{2} \mathrm{CVn}$ stars or ellipsoidal binaries, cannot be excluded. In a few stars we also revealed periodicities with periods shorter than $0.5 \mathrm{~d}$. Such periods are more typical for $\delta$ Scuti or $\beta$ Cephei-type 
variability. These stars are therefore good candidates for hybrid $\gamma$ Doradus $/ \delta$ Scuti stars similar to HD 8801 (Henry \& Fekel 2005). Another possibility is that they are hybrid SPB/ $\beta$ Cephei stars.

The periods, amplitudes and the position in the CMD are not sufficient to distinguish SPBs from $\gamma$ Doradus stars. Therefore we are going to gather UBV photometry and/or low-resolution spectroscopy for stars from our sample, which will allow us to improve the classification of these stars.

Acknowledgments. This work was supported by the MNil grant No. 1 P03D 01627 . The authors are grateful to the EC for the establishment of the European Helio- and Asteroseismology Network HELAS, which made their participation at this workshop possible.

\section{References}

Henry G. W., Fekel F. C., 2005, AJ, 129, 2026

Narwid A., Kołaczkowski Z., Pigulski A., 2006, Mem. Soc. Astron. Ital., 77, 342

Woźniak P. R., Udalski A., Szymański M., et al., 2002, Acta Astron., 52, 129 